Analyse des nationalsozialistischen und sowjetkommunistischen Herrschaftssystems nutzte und auf die zeitgenössischen Forschungsergebnisse zurückgriff.

\title{
4.5 Die Rolle der Totalitarismustheorie in der frühen Bundesrepublik
}

Nach dem Ausbruch des Ost-West-Konfliktes erwies sich die klassische Totalitarismustheorie (Arendt; Friedrich/Brzezinski) in den 1950er-Jahren als geeignetes Instrument, um die Westdeutschen im Kampf gegen den »neuen« totalitären Feind aus der Sowjetunion an die Seite der westlichen Staaten zu stellen sowie ein eindimensionales und personenzentriertes Bild des nationalsozialistischen Herrschaftssystems zu zeichnen, in dessen Zentrum Hitler stand. Anders gesagt: Die klassische Totalitarismustheorie besaß im Kalten Krieg in der Bundesrepublik insbesondere eine antikommunistische Funktion, die ein Grund dafür war, dass die Analyse des »Führerstaates« einen reduktionistischen Charakter aufwies.

Dabei konfrontierten bereits die 13 Nürnberger Prozesse die westdeutsche Nachkriegsgesellschaft nicht nur mit den nationalsozialistischen Massenverbrechen, sondern zeigten auch, dass das Unrechtsregime nicht auf eine kleine kriminelle Führungsclique um Hitler sowie die Herrschaftsinstitutionen der SS und Gestapo reduziert werden konnte. Gleichwohl beharrten die Vertreter der "Outlaw-Theorie« auf jener Vorstellung, in der der deutschen Bevölkerung der Platz eines »verführten Volkes« zugewiesen wurde, das für den begangenen Völkermord an den europäischen Juden und für die Kriegsverbrechen nicht zur Verantwortung gezogen werden dürfe. Zudem vertraten die deutschen Historiker und Publizisten die Auffassung, dass die »Hitler-Diktatur« einen Einbruch sowohl in der deutschen als auch der europäischen Geschichte darstellte, und betrachteten ihn als einen >Betriebsunfalk der Weltgeschichte, der sich zwar in Deutschland ereignete, allerdings in jedem Land möglich gewesen wäre. So stellten auch und vor allem Friedrich Meineckes einflussreicher Essay Die deutsche Katastrophe ${ }^{307}$ aus dem Jahre 1946 und - in stärkerem Maße - das zwei Jahre später erschienene Buch Europa und die deutsche Frage von Gerhard Ritter ${ }^{308}$ den Versuch dar, den Nationalsozialismus aus einer negativen gesamteuropäischen Entwicklung heraus zu erklären, die mit der Französischen Revolution ihren Anfang nahm und die parallel $\mathrm{zu}$ der grundsätzlich sgesunden existiert hätte. Das >Dritte Reich erschien als eine Katastrophe, die über Deutschland hereingebrochen war, und als ein historischer Zufall, der sich größtenteils auf den »Dämon« Hitler zurückführen ließ. Zudem setzten sich führende Historiker in Westdeutschland auch weniger mit der konkreten Geschichte des Nationalsozialismus auseinander als vielmehr mit dem Widerstand gegen das »Hitler-System«. In der bürgerlich-konservativen Opposition aus Offizieren, Kirchenvertretern und Beamten erkannten sie quasi ein »anderes Deutschland« und meinten insofern einen Ausweg aus der NS-Geschichte gefunden zu haben, als hiermit ein Anknüpfungspunkt für den

307 Friedrich Meinecke, Die deutsche Katastrophe, Wiesbaden 1946.

308 Gerhard Ritter, Europa und die deutsche Frage. Betrachtungen über die geschichtliche Eigenart des deutschen Staatsdenkens, München 1948. 
Neubeginn gefunden war. Somit avancierte der »20. Juli« zu einem überragenden Thema in der westdeutschen Geschichtswissenschaft der 1950er-Jahre. ${ }^{309}$

Vor dem Hintergrund des in der Bundesrepublik vorherrschenden Bildes der Geschichte des >Dritten Reiches $<$ und des einsetzenden Kalten Krieges war die klassische Totalitarismustheorie in der Bundesrepublik überaus populär. Sie ermöglichte einer substanziellen Auseinandersetzung mit dem nationalsozialistischen Herrschaftssystem und mit den begangenen Massenverbrechen aus dem Wege zu gehen, sodass für empirisch-differenzierte und komplexe Untersuchungen kein Platz war. Der Vergleich des Nationalsozialismus und des Sowjetkommunismus im Sinne der identifizierenden Totalitarismustheorie rückte bekanntlich weniger die Differenzen als die strukturellen Gemeinsamkeiten in den Blick und suchte sie in erster Linie in den Herrschaftssystemen. Fokussiert wurden der Terror und die massenkommunikativen Machtmittel, die sogenannte totalitäre Propaganda und chiliastische Ideologie, die Einparteienherrschaft und der Personenkult. »Vom NS-Staat«, so Peter Reichel, »konnte auf diese Weise das Bild eines monolithisch-totalitären Machtapparates entstehen, hierarchisch nach dem Führerprinzip durchorganisiert, ein gigantischer Lenkungsapparat, der die Massen zugleich umfassend ideologisierte, mobilisierte und kontrollierte, der sie führte und verführte. «110

Daran konnte auch Arendts Studie Elemente und Ursprünge totaler Herrschaft nichts ändern. Unter Ausblendung zentraler Untersuchungsteile und -ergebnisse konnte wie erwähnt so ein simplifizierendes und dämonisierendes Bild des Nationalsozialismus gezeichnet werden, indem sowohl die komplexe Vorgeschichte des >Dritten Reiches als auch die spezifische Strukturlosigkeit des Behemoth (Neumann) unberücksichtigt blieb. Das nationalsozialistische Herrschaftssystem wurde auf den »Führer« reduziert, der durch die Geheimpolizei und neuartige Mittel und Techniken sowie durch "Ideologie und Terror« seine Herrschaft ausübte und sicherte. Als ebenso folgenreich erwies sich die Tatsache, dass auch Arendts Totalitarismusstudie in der frühen Bundesrepublik angesichts des Kalten Krieges die Möglichkeit bot, den Nationalsozialismus und den Sowjetkommunismus als wesensgleiche Herrschaftssysteme darzustellen. Die Vorstellung, wonach die sbraune mit der sroten Diktatur umstandslos gleichgesetzt werden könnte, fiel vor allem deshalb auf fruchtbaren Boden, weil es politisch-ideologischen Bedürfnissen entgegenkam. Nachdem das NS-Regime untergegangen war, bezog sich der Totalitarismusbegriff aktuell politisch in erster Linie auf das kommunistische Regime. Auf diesem Weg konnte die identifizierende Totalitarismustheorie im Zeichen des Antikommunismus zur Integrationsideologie des neuen Staates werden. Mit anderen Worten: Die klassische Totalitarismustheorie wurde vor allem für antikommunistische politische Zwecke instrumentalisiert. Sie diente nicht nur als außerstaatliche Feinderklärung, sondern beeinflusste vor allem auch das innenpolitische Klima, indem nicht nur die politischen Kräfte als »fünfte Kolonne« Moskaus stigmatisiert wurden, die eine »Veränderung der Eigentumsverhältnisse und

309 Vgl. exemplarisch bes. Hans Rothfels, Die deutsche Opposition gegen Hitler. Eine Würdigung, Krefeld 1951.

310 Peter Reichel, Zwischen Dämonisierung und Verharmlosung: Das NS-Bild und seine politische Funktion in den 5oer-Jahren. Eine Skizze, in: Axel Schildt/Arnold Sywottek (Hg.), Modernisierung im Wiederaufbau: die westdeutsche Gesellschaft der 5oer-Jahre, Bonn 1993, S. 679-692, hier S. 685. 
der Wirtschaftsordnung « forderten. ${ }^{311}$ Überhaupt wurde in diesem Zusammenhang nahezu jede prinzipielle Kritik, die von der Linken und der (linken) außerparlamentarischen Oppositionsbewegung an der sfreiheitlich-demokratischen Grundordnung, sowie der Bundesregierung und ihrer Politik formuliert wurde, mit dem Kommunismusvorwurf konfrontiert. ${ }^{312}$

Allerdings konnte sich durch die antikommunistische bzw. antibolschewistische Ausrichtung ein zentraler Eckpfeiler der NS-Ideologie behaupten und mithin politisch relegitimieren. Nach der Berlinblockade 1948/49 und insbesondere nach dem Ausbruch des Koreakrieges im Juni 1950 und der danach in Gang kommenden Wiederbewaffnung wurde von ebendieser Feindideologie immer häufiger Gebrauch gemacht, als durch den amerikanischen Feldzug gegen den »Bolschewismus« nicht nur ehemalige Wehrmachtssoldaten, sondern viele Westdeutsche glauben konnten, zumindest in dieser Hinsicht seit Langem auf der richtigen Seite gestanden zu haben. So konnte es auch nicht verwundern, dass im Grunde genommen die Totalitarismustheorie prädestiniert war, um im Laufe der Jahre mit der bundesdeutschen Verfassung politisch deckungsgleich werden konnte: Einerseits konnten mit ihr ex negativo die Prinzipien der parlamentarischen Demokratie und des liberalen Rechtsstaates propagiert werden und anderseits der Kampf gegenüber dem sowjetischen Kommunismus einschließlich seiner Satellitenstaaten legitimiert werden. Der von der Regierung und fast aller im Bundestag vertretenen Parteien immer wieder beschworene »antitotalitäre« Konsens wies hierbei allerdings die angesprochene politisch überaus problematische Dimension auf, denn, so der Hamburger Politikwissenschaftler und Historiker Wolfgang Kraushaar:

Antifaschistisch war der Antitotalitarismus vor allem der Vergangenheit gegenüber gemeint, antikommunistisch aber vor allem gegenüber der Cegenwart. Die Rechts-LinksSymmetrie wurde mit dem Verbot der Sozialistischen Reichspartei (SRP) 1952 und dem der KPD 1956 scheinbar gewahrt, in Wirklichkeit jedoch hatte, was sich an der Strafverfolgung extremistischer Gruppierungen zeigte, die Totalitarismusdoktrin in der Bundesrepublik Schlagseite: Es war sehr viel riskanter, eine als kommunistisch verdächtige Position einzunehmen als eine nazistische. ${ }^{313}$

So wurde die "politische Justiz« $\mathrm{zu}$ einem zentralen Instrument, mit dem Personen bekämpft wurden, die des Kommunismus verdächtigt wurden oder nicht bereit waren, sich von ihrer kommunistischen Überzeugung zu distanzieren. ${ }^{314}$

Der in der frühen Bundesrepublik immer wieder beschworene Antitotalitarismus verknüpfte also die Distanzierung vom Nationalsozialismus angesichts des »drohen-

311 Christoph Kleßmann, Die doppelte Staatsgründung. Deutsche Geschichte 1945-1955, 4., erg. Aufl., Bonn 1986, S. $256 \mathrm{f}$.

312 Vgl. Christoph Kleßmann, Zwei Staaten, eine Nation. Deutsche Geschichte 1955-1970, Bonn 1988, S. 59.

313 Wolfgang Kraushaar, Spuren eines Paradigmenwechsels. Von der Totalitarismus- zur Faschismustheorie, in: Ders., Linke Geisterfahrer. Denkanstöße für eine antitotalitäre Linke, Frankfurt a. M. 2001, S. 109-130, hier S. 128.

314 Vgl. hierzu Alexander Brünneck, Politische Justiz gegen Kommunisten in der Bundesrepublik Deutschland 1949-1969, Frankfurt a. M. 1978. 
den Bolschewismus« geschickt mit dem außen- und innenpolitischen Kampf gegen den (sowjetischen) Kommunismus. Für die westdeutsche Wiederaufbaugesellschaft, in die sich die ehemalige »Volksgemeinschaft« verwandelte, hatte dies zweifelsohne den politischen Vorteil, dass sie von der Auseinandersetzung mit der NS-Geschichte zum Teil entlastet wurde. Mehr noch: Im Fahrwasser des antikommunistischen Zeitgeistes und schnellen Überganges zum Kalten Krieg endete die Diskussion um die »deutsche Schuldfrage u und mithin die Thematisierung der individuellen NS-Vergangenheit. Zudem regte sich im Zuge der sich ausbreitenden »Schlussstrichmentalität« auch mehr und mehr Widerstand gegen die Entnazifizierungspolitik. Denn, so Peter Reichel:

Die offensichtlichen Mängel und Ungerechtigkeiten, die mit diesen historisch einmaligen, bürokratischen und gerichtlichen Verfahren zwangsläufig einhergingen, wurden jedoch in der deutschen Bevölkerung vielfach als neues Unrecht und Schuldentlastung betrachtet. Kampagnen für die in alliierten Cefängnissen einsitzenden `Kriegsverbrecher und gegen die, auch von den Parteien und Kirchen vehement verurteilte Entnazifizierung gaben dem teilweise aggressiven Ausdruck. ${ }^{315}$

In diesem Zusammenhang gebärdeten sich die Westdeutschen in den 1950er-Jahren im Grunde genommen wie eine nur »schwach säkularisierte Volksgemeinschaft «, ${ }^{316}$ die genau wusste, dass das NS-Regime keinesfalls nur auf die kleine kriminelle Führungsclique um den »Dämon" Hitler sowie die Herrschaftsinstitutionen der SS und die Gestapo reduziert werden konnte. Auch aus diesem Grund war die Totalitarismustheorie nicht nur in der Wissenschaft in der frühen Bundesrepublik so populär, weil sie durch einen einseitigen und instrumentellen Blick ebendieses NS-Bild bestätigte und zudem die Möglichkeit bot, das deutsche Volk als politisch verführtes darzustellen. Die nationalsozialistischen Massenverbrechen im Allgemeinen und die Vernichtung der europäischen Judentum im Besonderen wurden hierbei nicht geleugnet, spielten allerdings in der konkreten Auseinandersetzung mit der jüngsten Geschichte keine Rolle und wurden verdrängt. Insbesondere durch die vorherrschende Überbetonung der Rolle Hitlers konnten die diplomatischen, militärischen, bürokratischen, medizinischen, wirtschaftlichen, kulturellen und bildungsbürgerlichen Eliten ihre Mitverantwortung und Mitwirkung in das verbrecherische System kaschieren. Dies konnte auch deshalb gelingen, weil auch die Totalitarismustheorie, mit dem Verweis auf ein totalitäres Herrschaftssystem, das bereits vor und nach dem NS-System existierte, ihnen einen legitimatorischen Umhang verschaffte. Gleichzeitig ermöglichte es ihnen die politische und soziale Integration in die prosperierende westdeutsche Wiederaufbaugesellschaft der 1950er-Jahre.

315 Reichel, Vergangenheitsbewältigung in Deutschland, S. 18.

316 Frei, Vergangenheitspolitik, S. 399. 


\section{Der »Kongreß für kulturelle Freiheit» in Berlin}

Der in Westberlin vom 26. bis 30. Juni 1950 veranstaltete internationale »Kongreß für kulturelle Freiheit« war eine Reaktion der westlichen Welt auf die kommunistische Weltfriedensbewegung. Mit der unter der Führung von amerikanischen Regierungsstellen neu geschaffenen Organisation sollte eine antikommunistische Dauerkampagne institutionalisiert werden. Der Sowjetunion war es nämlich besonders seit 1948/49 immer mehr gelungen, die Friedensthematik für die kommunistische Propaganda zu nutzen. Unter anderem mit einer Serie von kleinen und größeren Konferenzen bzw. Kongressen hatten sie es erreicht, die Friedensthematik im öffentlichen Bewusstsein zu verankern. In der Tradition der Agitations- und Propagandamethoden des Münzenberg-Apparates stehend, wurden hierbei unter der Ägide der Kominform vor allem westliche Intellektuelle als "fellow travellers« eingebunden. So entstand ein breit gestreutes Bündnis von Anhängern der Sowjetunion, das von Parteigängern des Kommunismus bis zu Vertretern aus der Kirche und dem bürgerlich-humanistischen Lager reichte. Nach Auffassung vieler Schriftsteller, Künstler und Wissenschaftler galt die Sowjetunion unter Stalin als Hüterin des Weltfriedens, obwohl sie nach dem Ende des Zweiten Weltkrieges ein imperialistisches System in Osteuropa errichtet hatte. Der Sowjetunion war es angesichts der tief sitzenden Erinnerungen an die Schrecken des Zweiten Weltkrieges und der zeitgenössischen Drohung einer atomaren Vernichtung gelungen, sich an die Spitze einer von Millionen von Menschen getragenen weltweiten Friedensbewegung zu stellen, ohne dass die Infiltrierung und diskrete Steuerung ebendieser Massenbewegung durch die Stalinisten den Beteiligten unmittelbar einsichtig wurde. Das galt insbesondere für Frankreich mit seiner überaus einflussreichen kommunistischen Partei, wo es die Sowjetunion vor dem Hintergrund der schdanowschen Zweiweltentheorie schaffte, die Friedensthematik mit dem tiefsitzenden antiamerikanischen Ressentiment zu verbinden. Auf der anderen Seite waren die Vereinigten Staaten nach dem Abwurf der Atombomben über Hiroshima und Nagasaki mit dem Makel behaftet, auf eine Politik der Hochrüstung und der Stärke zu setzen, die angesichts von einflussreichen amerikanischen Stimmen, im Kalten Krieg eine umfassende aggressive antisowjetische Politik einzuschlagen, neue Nahrung bekam. ${ }^{317}$ Daraus folgte für die Situation Ende der 1940er-Jahre, dass sich die Vereinigten Staaten vor allem ideologisch offensichtlich in der Defensive befanden, was nicht zuletzt durch die kommunistische Weltfriedensbewegung verursacht wurde.

Seit März 1949 nahm die Idee für den internationalen »Kongreß für kulturelle Freiheit« in Berlin langsam Gestalt an. Konkreter Anlass war die in direkter Fortsetzung des Breslauer Friedenskongresses (August 1948) stehende »Cultural and Scientific Conference for World Peace« in New York. Der Ende März 1949 stattgefundene Kongress war ein »heimliches" Vorhaben der Kominform, der sich gegen die bevorstehende Gründung der NATO wandte und als zentrales Thema die kulturelle Freiheit in den Mittelpunkt rückte. Die Redner, unter denen sich auch der ehemalige amerikanische Vizepräsident und »fellow-traveller « Henry Wallace befand, gingen hierbei allerdings nicht auf die totalitäre Praxis des sowjetischen Staates ein, denen Wissenschaftler,

317 Vgl. für diese poltische Konzeption bes. James Burnham, The Struggle for the World, New York 1947 sowie ders., The Coming Defeat of Communism, New York 1950 (dt. Ausg.: Die Strategie des Kalten Krieges, Stuttgart 1950). 